\title{
On the Alpha-Power Inverse Weibull Distribution
}

\author{
Dina A. Ramadan \\ Mathematics Department, Faculty of Science, \\ Mansoura University, Mansoura, Egypt
}

\author{
Walaa Magdy A. \\ Mathematics Department, Faculty of Science, \\ Mansoura University, Mansoura, Egypt
}

\begin{abstract}
A new method for adding parameters to a well-established distribution to obtain more flexible new families of distributions is applied to the inverse Weibull distribution (IWD). This method is known by the Alpha-Power transformation (APT) and introduced by Mahdavi and Kundu [9]. The statistical and reliability properties of the proposed models are studied. The estimation of the model parameters by maximum likelihood and the observed information matrix are also discussed. The extended model is applied on a real data and the results are given and compared to other models.
\end{abstract}

\section{Keywords}

Weibull distribution; inverse Weibull distribution; maximumlikelihood estimation; survival function; fisher information matrix; order statistic.

\section{INTRODUCTION}

Reliability is a human characteristic that has been in.ect for a long period of time. Reliability acts the probability of components parts and systems to perform the desired task for a specific period of time without failure in fixed environments with required confidence. The Exponential, Raylight, linear failure rate, Weibull and inverse Weibull are the most common life distribution in reliability and life testing [11]. Exponential distribution has constant failure rate distribution, Raylight distribution has increasing failure rate distribution, and there are distributions that have increasing or decreasing failure rate introduced by Nassar and Abo-kasem [10]. The hazard rate function can be of bath tube shape it plays a central role in the work of reliability engineers (the Weibull distribution, Generalized exponential, Rraylight distribution, inverse Weibull) [4]. The inverse Weibull was originally used in reliability industry, it is one of the most common life distribution in reliability engineering, the need for an extended form of the distribution grow in many applied fields Corderio and Lemonte[7]. If the random variable $Y$ has a Weibull distribution, then the random variable $X=Y^{-1}$ has an inverse Weibull distribution with cumulative distribution function (cdf)

$$
F(x)=e^{-\lambda x^{-\beta}}
$$

The probability density function (pdf)

$$
f(x)=\lambda \beta \mathrm{x}^{-(\beta+1) \mathrm{e}^{-\lambda \mathrm{x}-\beta},}
$$

And hazard rate function

$$
h(x)=\lambda \beta \mathrm{x}^{-(\beta+1)}\left(\mathrm{e}^{-\lambda \mathrm{x}^{-\beta}}-1\right)^{-1} .
$$

The inverse Weibull is more suitable than the Weibull distribution because the inverse Weibull distribution gives satisfactory parametric fit if the data indicate anon monotone and unimodal hazard rate function. The hazard rate function of the IW can be an increase or decrease depending on the value of the shape parameter. The IW distribution is useful to modal several data such as the time to fall of an isolated liquid subjected to the work of a fixed stress and decay of mechanical components such as compressor and crankshafts of diesel engines introduced by Nassar and abo-kasem [10]. Adding parameters to a well-known distribution helps us to obtain more flexible new families of distribution [2]. Madhdavi and kundu [9] suggest an interesting method for generating distribution function as follows:

Let $F(x)$ be the CDF of a continuous random variable $X$, then $\alpha$ - power transformation of $F(x)$ for $X \in R$, is defined as follows:

$$
F_{A P T}=\left\{\begin{array}{cc}
\frac{\alpha^{F(x)-1}}{\alpha-1} & , \alpha>0, \alpha \neq 1 \\
F(x) & , \alpha=1 .
\end{array}\right.
$$

If $f(x)$ is an absolute continuous distribution function with the probability density function $f(x)$ then $F_{A P T}$ is also an absolute continuous distribution function with the pdf

$$
f_{A P T}=\left\{\begin{array}{cl}
\frac{\log \alpha}{\alpha-1} f(x) \alpha^{F(x)}, & \alpha>0, \alpha \neq 1 \\
f(x), & \alpha=1 .
\end{array}\right.
$$

The survival reliability function $S_{A P T}$ and the hazard rate function $h_{A P T}$ for APT distribution are in the following forms

$$
S_{A P T}=\left\{\begin{array}{c}
-\frac{\alpha}{\alpha-1}\left(1-\alpha^{\mathrm{F}(\mathrm{x})-1}\right), \alpha>0, \alpha \neq 1 \\
1-\mathrm{F}(\mathrm{x}), \quad \alpha=1 .
\end{array}\right.
$$

and

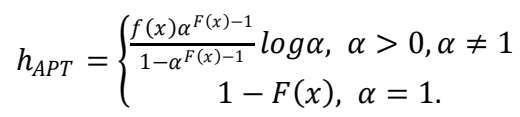

The rest of the paper is organized as follows. Section 2 introduces alpha power inverse Weibull (APIW) distribution. The structural characteristics of APIW distribution including the behavior of the probability density function, the hazard rate function, the reversed hazard rate function, the (reversed) residual life, the moments and the associated moments, quantile function and finally the order statistics and extreme values are studied in sections $3,4,5,6$. Section 7 concerns with the Maximum likelihood estimators of the unknown parameters and some inferential issues. Simulation schemes are obtained in section 8. Finally, a real data life application has illustrated the potential of APIW distribution compared with other distributions in section 9 .

\section{THE ALPHA POWER INVERSE WEIBULL DISTRIBUTION(APIW)}

In this section, we apply APT method to apeci.c class of distribution function, namely to an inverse Weibull distribution, and named this new distribution the threeparameter APIW distribution. The CDF and PDF of the inverse Weibull distribution is

$F(x, \lambda, \beta)=e^{-\lambda x^{-\beta}}, x>0, \lambda, \beta>0$, 
$f(x, \lambda, \beta)=\lambda \beta x^{-(\beta+1)} e^{-\lambda x^{-\beta}} \quad, x>0, \lambda, \beta>0$.

where $\lambda$ is the scale parameter and $\alpha, \beta$ is a shape parameter. Then the CDF and PDF of Alpha-power-inversed Weibull Distribution are

$$
F(x, \alpha, \beta, \lambda)= \begin{cases}\frac{\alpha^{\mathrm{e}^{-\lambda x^{-\beta}}-1}}{\alpha-1}, & \alpha \neq 1 \\ \mathrm{e}^{-\lambda x^{-\beta}}, & \alpha=1\end{cases}
$$

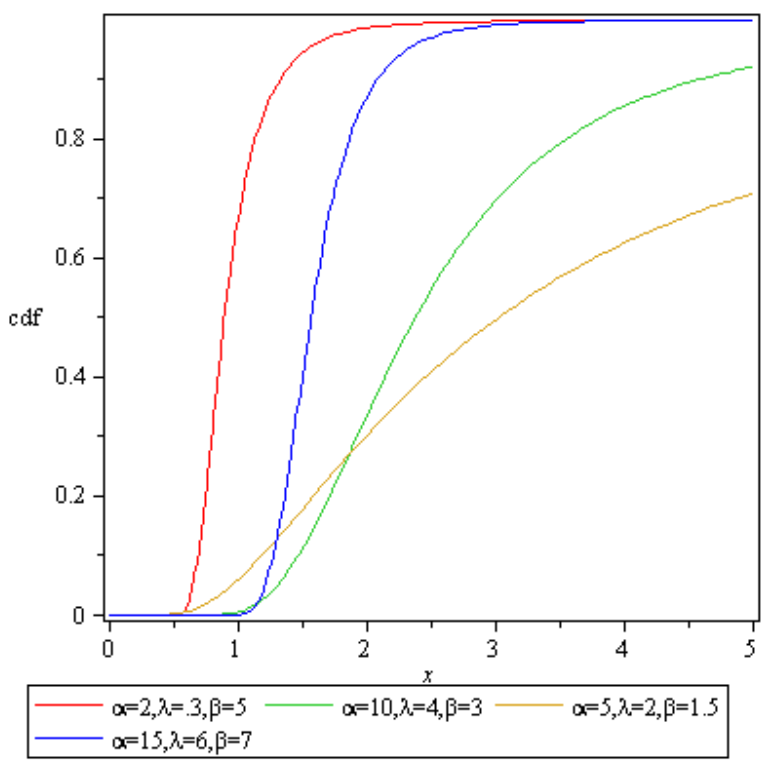

Fig.1. plot of the cdf of APIW, at $\alpha \neq 0$.

and

$f(x, \alpha, \beta, \lambda)=$
$\left\{\begin{array}{c}\frac{\log \alpha}{\alpha-1} \lambda \beta x^{-(\beta+1)} e^{-\lambda x^{-\beta}} \alpha^{e^{-\lambda x-\beta}}, \alpha \neq 1, x, \lambda, \alpha, \beta>0 \\ \lambda \beta x^{-(\beta+1)} e^{-\lambda x^{-\beta}}, \alpha=1, x, \lambda, \beta>0 .\end{array}\right.$

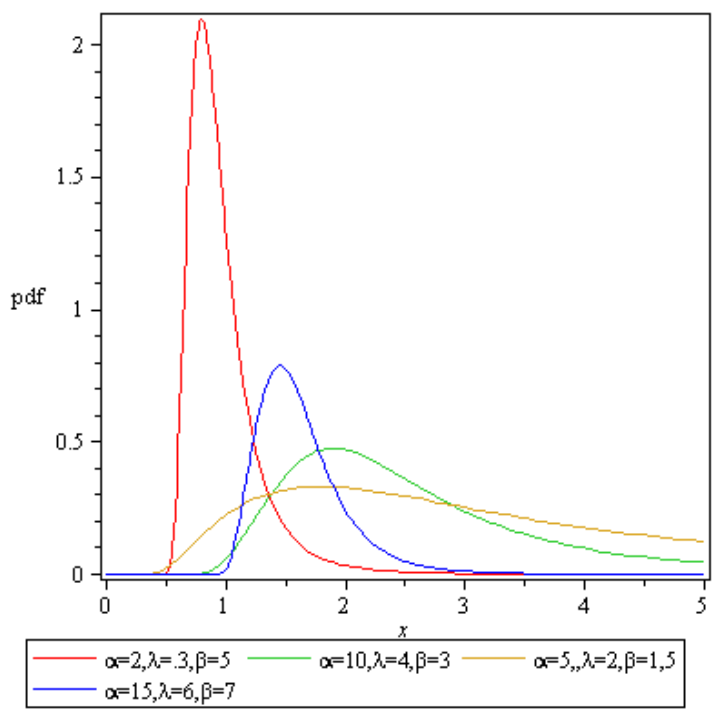

Fig.2.The pdf of APIW, $\alpha \neq 0$.

Where $\beta$ is shape parameter and $\lambda$ is the scale parameter.

The survival reliability function $S_{A P I W}$ for APIW distribution is given by
$S_{\mathrm{APIW}}= \begin{cases}\frac{\alpha}{\alpha-1}\left(1-\alpha \mathrm{e}^{-\lambda x^{-\beta}}-1\right), & \alpha \neq 1 \\ 1-\mathrm{e}^{-\lambda \mathrm{x}^{-\beta}}, & \alpha=1 .\end{cases}$

Hence the graph for some values of the parameters is given as follows:

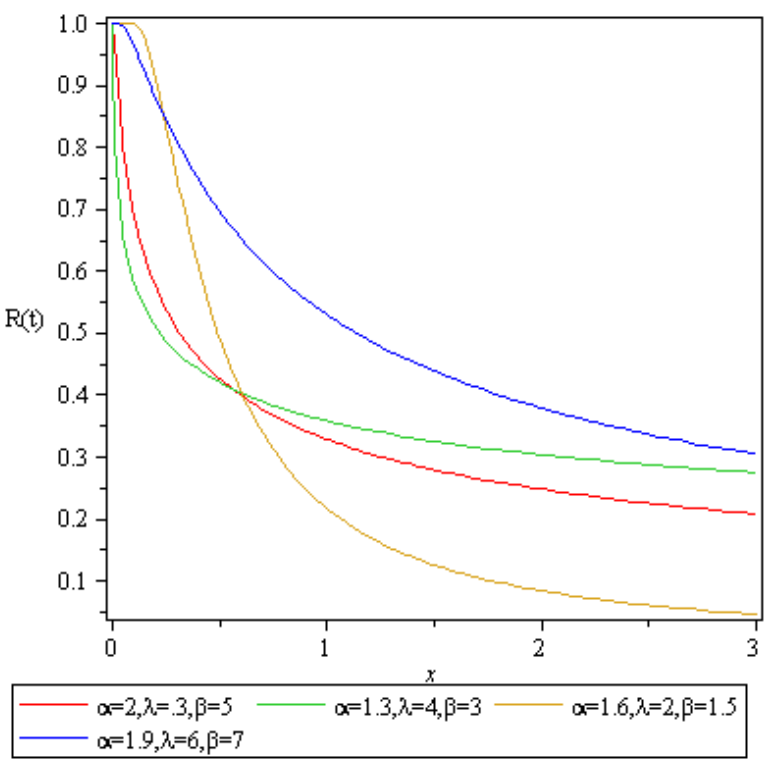

Fig.3 the reliability function of APIW, $\alpha \neq 0$.

\section{STRUCTURAL CHARACTERISICS}

In this section, we study the structural characteristics for APIW distribution.

In particular, if $\mathrm{x} \sim \mathrm{APIW}(\alpha, \beta, \lambda)$ then the functional behavior of the density function ad of the hazard rate function, mean residual life function and others are derived and studied in details.

The behavior of the probability density function of Alpha Power Inverse Weibull distribution

\section{Theorem 1:}

The pdf of alpha power inverse Weibull is:

(i) Decreasing if $\lambda>0, \beta \leq 1$,

(ii) Unimodal if $\beta<-1, \lambda<0$.

Proof

(i) since

$$
\begin{gathered}
\ln f(x)=\ln (\log \alpha)- \\
-\ln (\alpha-1)+\ln (\lambda \beta) \\
-(\beta+1) \ln (x)-\lambda x^{-\beta} \\
+e^{-\lambda x^{-\beta} \ln (\alpha),} \\
\frac{d}{d x} \ln f=\frac{-\beta-1}{\mathrm{x}}+\lambda \beta x^{-\beta-1}\left[1+e^{-\lambda x^{-\beta}} \ln (\alpha)\right], \\
\frac{\mathbf{d}}{\mathbf{d} \mathbf{x}} \ln \mathbf{x}(\mathbf{x})<\mathbf{0} \text {, for } \lambda>0, \beta \leq-1
\end{gathered}
$$

Then $\mathrm{F}(\mathrm{x})$ is decreasing

(ii) If $\beta<-1, \lambda<0$ since

$$
\begin{array}{r}
\frac{\mathrm{d}^{2}}{\mathrm{dx}^{2}} \ln (\mathrm{f}(\mathrm{x}))=\frac{\beta+1}{\mathrm{x}^{2}}-\lambda \beta(\beta+1) \mathrm{x}^{-\beta-2}-((\lambda+1) \beta \\
+1) \lambda \beta \mathrm{x}^{-\beta-2} \mathrm{e}^{-\lambda \mathrm{x}^{-\beta}} \ln (\alpha)<0
\end{array}
$$


Hence $f(x)$ has a local maximum at $x_{0}$. The behavior of APIW distribution density can be illustrated as in Fig.1.

\section{HAZARED RATE FUNCTION}

The hazard rate function of APIW is given by

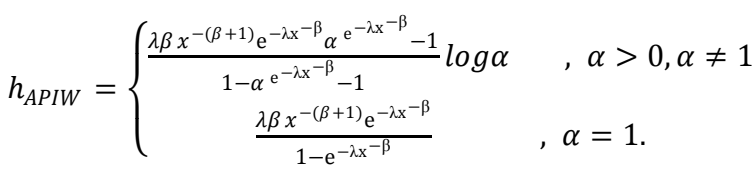

Theorem 2: The hazard rate function of alpha power inverse Weibull distribution is

(i) Increasing if $\lambda, \beta>0$

(ii) Decreasing Hazard rate if $\lambda>0, \beta \geq-1$

\section{Proof}

(i) Since,

$$
\begin{aligned}
& \frac{d}{d x} h(x)=\frac{\lambda \beta x^{-(\beta+1)} \mathrm{e}^{-\lambda x^{-\beta}} \alpha^{\mathrm{e}^{-\lambda x}-\beta}-1 \ln (\alpha)}{x\left(1-\alpha \mathrm{e}^{-\lambda x^{-\beta}}-1\right)}+ \\
& {\left[-(\beta+1)+\lambda \beta x^{-\beta}+2 x^{-\beta} e^{-\lambda x^{-\beta}}+\right.} \\
& \left.\frac{2 \mathrm{x}^{-\beta} \mathrm{e}^{-\lambda \mathrm{x}-\beta_{\alpha} \mathrm{e}^{-\lambda \mathrm{x}-\beta}-1}}{1-\alpha \mathrm{e}^{-\lambda \mathrm{x}-\beta}-1}\right] \text {, }
\end{aligned}
$$

for $\lambda, \beta>0, \frac{d}{d x} h(x)>0$ then hazard rate function is increasing.

(ii) for $\lambda>0, \beta>-1, \frac{d}{d x} h(x)>0$ then hazard rate function is decreasing.

HRF of the APIW distribution are displayed in Fig.4 for different values of $\alpha, \beta$ and $\lambda$.

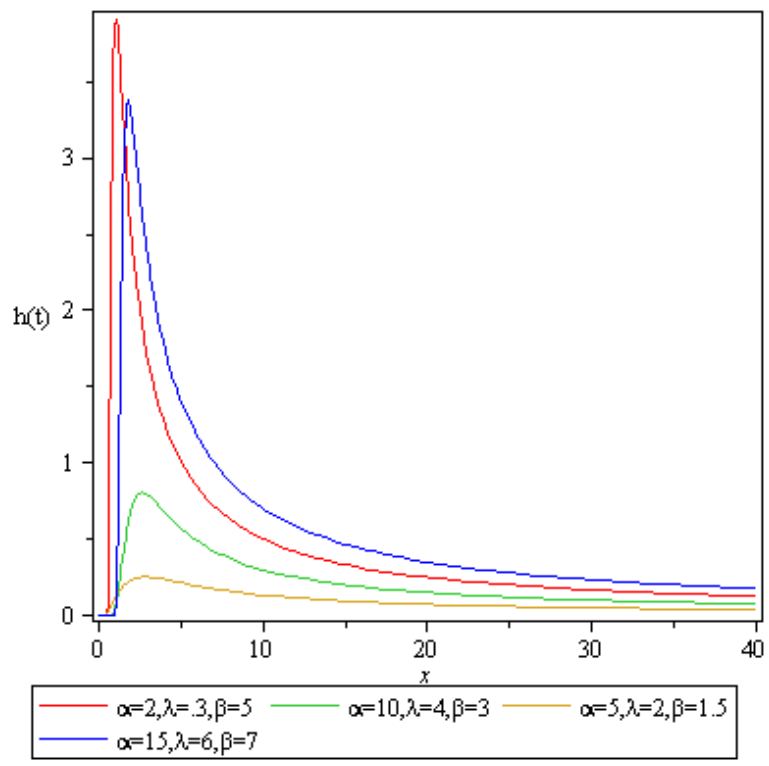

Fig.4. The hazard rate function of APIW, $\alpha \neq 0$.

\subsection{Reversed Hazard Rate}

The reserved hazard rate can be de.ned as the conditional random variable $[t-x / x \leq t]$ denote the time elapsed from the failure of a component given that its life is less than or equal this random variable is called also the time since.

$$
r(x)=\frac{f(x)}{F(x)}
$$

then

$$
\begin{gathered}
r(x)=\frac{\lambda \beta x^{-(\beta+1)} \mathrm{e}^{-\lambda x^{-\beta}} \alpha^{\mathrm{e}^{-\lambda x-\beta}}}{\alpha^{\mathrm{e}^{-\lambda x^{-\beta}}-1}} \log \alpha, \\
x>0, \alpha \neq 1, \alpha, \beta, \lambda>0
\end{gathered}
$$

\section{2 (Reversed) Residual Life Function}

Residual life and reversed residual life random variables are used extensively in risk analysis. Accordingly, we investigate some related statistical functions, such as survival function, mean and variance in connection with APIW distribution.

The residual life is described by the conditional random variable $\mathrm{R}(\mathrm{T})=\mathrm{x}-\mathrm{t} / \mathrm{x}>t, t \geq 0$, and defined as the period from time $t$ until the time of failure.

Analogously, the reversed residual life can be defined as $\bar{R}(t)=\mathrm{t}-\frac{\mathrm{x}}{\mathrm{x}} \leq t, t \geq 0$, which denotes the time elapsed from the failure of a component given that its life $\leq \mathrm{t}$.

i. Residual lifetime function

The survival function of a residual lifetime $S(t), t \geq 0$, for APIW distribution is given by

$$
\mathrm{S}_{\mathrm{R}(\mathrm{t})}(x)=\left(1-\alpha^{\mathrm{e}^{-\lambda(\mathrm{x}+\mathrm{t})^{-\beta}}-1}\right)\left(1-\alpha^{\mathrm{e}^{-\lambda(\mathrm{x})^{-\beta}}-1}\right)^{-1}
$$

and its PDF is

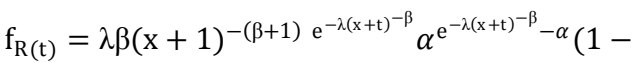
$\left.\alpha^{\mathrm{e}^{-\lambda(\mathrm{x}+\mathrm{t})^{-\beta}}-1}\right)\left(1-\alpha^{\mathrm{e}^{-\lambda(\mathrm{x})^{-\beta}}-1}\right)^{-1} \log \alpha$.

Consequently, the hazard rate function of $R(t)$ has the following form

$$
\mathrm{h}_{\mathrm{R}(\mathrm{t})}(x)=\lambda \beta(\mathrm{x}+1)^{-(\beta+1) \mathrm{e}^{-\lambda(\mathrm{x}+\mathrm{t})^{-\beta}}} \alpha^{\mathrm{e}^{-\lambda(\mathrm{x}+\mathrm{t})^{-\beta}-1} \log \alpha .}
$$

\section{ii. Reversed residual life function}

The survival function of the reversed residual lifetime $\bar{R}(t)$ for APIW Distribution is given by

$$
S_{\bar{R}(t)}(\mathrm{x})=\frac{\mathrm{F}(\mathrm{x}+\mathrm{t})}{\mathrm{F}(\mathrm{t})}=\frac{\alpha^{\mathrm{e}^{-\lambda((\mathrm{t}-\mathrm{x}))^{-\beta}}-1}}{\alpha^{\mathrm{e}^{-\lambda \mathrm{t}^{-\beta}}-1}}, 0<x \leq t,
$$

Hence the probability density function of $\bar{R}(\mathrm{t})$ takes the following form

$$
f_{\bar{R}(t)}(\mathbf{x})=-\frac{\lambda \beta(\mathrm{t}-\mathrm{x})^{-\beta} \mathrm{e}^{-\lambda((\mathrm{t}-\mathrm{x}))^{-\beta}} \alpha^{\mathrm{e}^{-\lambda((\mathrm{t}-\mathrm{x}))^{-\beta}}} \ln \alpha}{\alpha^{\mathrm{e}^{-\lambda \mathrm{t}^{-\beta}}-1}} .
$$

Consequently, the hazard rate function of the reversed residual lifetime $\bar{R}(t)$ has the following form

$$
h_{\bar{R}(t)}(\mathbf{x})=\frac{\lambda \beta(\mathrm{t}-\mathrm{x})^{-\beta} \mathrm{e}^{-\lambda((\mathrm{t}-\mathrm{x}))^{-\beta}} \alpha^{\mathrm{e}^{-\lambda((\mathrm{t}-\mathrm{x}))^{-\beta}}} \ln \alpha}{1-\alpha^{\mathrm{e}^{-\lambda \mathrm{t}^{-\beta}}}} .
$$

\section{STATISTICAL INFERENCE}

\subsection{Moments}

Let $\mathrm{x} \sim A P I W(\alpha, \beta, \lambda)$. Hence, one can easily get the $r^{\text {th }}$ moment as follows:

$$
\mu_{r}=\int_{0}^{\infty} x^{r} f_{A P I W}(x) d x .
$$

Using the series representation

$$
\alpha^{-y}=\sum_{k=0}^{\infty} \frac{(-\log \alpha)}{k !} y^{k},
$$


We then get the $r^{\text {th }}$ moment of $\mathrm{x}$

$$
=\left\{\begin{array}{cc}
\sum_{k=0}^{\infty} \frac{\left.(\log \alpha)^{k+1}(k+1)^{\frac{r}{\beta}}-1\right)}{(1-\alpha) k !} \lambda^{\frac{r}{\beta}} \Gamma\left(\frac{-r}{\beta}+1\right), \alpha>0, \alpha \neq 1 \\
-\lambda^{\frac{r}{\beta}} \Gamma\left(\frac{-r}{\beta}+1\right) & , \alpha=1
\end{array}\right.
$$

Thus, the mean and the variance of APIW are respectively given as follows

$$
\begin{aligned}
& E(x) \\
& =\left\{\begin{array}{cc}
\sum_{k=0}^{\infty} \frac{\left.(\log \alpha)^{k+1}(k+1)^{\frac{1}{\beta}}-1\right)}{(1-\alpha) k !} \lambda^{\frac{1}{\beta}} \Gamma\left(\frac{-1}{\beta}+1\right), \alpha>0, \alpha \neq 1 \\
-\lambda^{\frac{1}{\beta}} \Gamma\left(\frac{-1}{\beta}+1\right) & , \alpha=1
\end{array}\right.
\end{aligned}
$$

and

$$
=\left\{\begin{array}{c}
\sum_{\mathrm{k}=0}^{\infty} \frac{(\log \alpha)^{\mathrm{k}+1}(\mathrm{k}+1)^{\frac{2}{\beta^{\beta}}-1}}{(1-\alpha) \mathrm{k} !} \lambda^{\frac{2}{\beta}} \Gamma\left(\frac{-2}{\beta}+1\right)- \\
{\left[\sum_{\mathrm{k}=0}^{\infty} \frac{(\log \alpha)^{\mathrm{k}+1}(\mathrm{k}+1)^{\frac{1}{\beta}-1}}{(1-\alpha) \mathrm{k} !} \lambda^{\frac{1}{\beta}} \Gamma\left(\frac{-1}{\beta}+1\right)\right]^{2}, \alpha \neq 1} \\
-\lambda^{\frac{2}{\beta}}\left[\Gamma\left(-\frac{2}{\beta}+1\right)+\left(\Gamma\left(\frac{-1+\beta}{2}\right)\right)^{2}\right], \alpha=1 .
\end{array}\right.
$$

The moment generating a function of $\operatorname{APIW}(\alpha, \beta, \lambda)$ can be obtained as

$$
\begin{aligned}
M_{X}(t) & =\mathrm{E}\left(\mathrm{e}^{\mathrm{tx}}\right)=\int_{0}^{\infty} e^{t x} \mathrm{f}(\mathrm{x}) \mathrm{dx} \\
& =\sum_{r=0}^{\infty} \frac{t^{r}}{r !} \int_{0}^{\infty} x^{r} f(x) d x
\end{aligned}
$$

\subsection{Quantile}

The $q^{\text {th }}$ quantile $x_{q}$ of $F_{A P I W}(x)$ for $\alpha \neq 1$ can be obtained as $Q_{x}(x)=\inf \{\mathrm{x} \in R: p \leq F(x)\}$, by using the transformation $f(q)=q$.

Then the $q^{\text {th }}$ quantile of $\operatorname{APIW}(\alpha, \beta, \lambda)$ is given by

$$
F\left(x_{q}\right)=q,
$$

$\mathrm{x}_{\mathrm{q}}=-\lambda^{\frac{1}{\beta}}\left[\log \frac{\log [1+(\alpha-1) q}{\log \alpha}\right]^{\frac{-1}{\beta}}$.

At $\mathrm{q}=0.5$, the $\mathrm{q}^{\text {th }}$ quantile reduced to the median which is given by

$$
\mathrm{x}_{0.5}=-\lambda^{\frac{1}{\beta}}\left[\log \frac{\log 11+(\alpha-1) 0.5}{\log \alpha}\right]^{\frac{-1}{\beta}} .
$$

\section{ORDER STATISTICS}

The order statistics have various applications in reliability and life testing. The order statistics arise in the study of the reliability of a system.

Let $X_{1}, X_{2}, \ldots . X_{n}$ be a simple random sample from $\operatorname{APIW}(\alpha$ $, \beta, \lambda)$ with cumulative distribution function and pdf in $\mathrm{Eq}(10), \mathrm{Eq}(11)$ respectively. Let $X_{(1: n)}, X_{(2: n)} \ldots \ldots \ldots X_{(n: n)}$ denote the order statistics obtained from this sample.
In reliability literature, $X_{(I: n)}$ denote the lifetime of an $(n-i+1)-$ out of $-n$ system that consists of $n$ independent and identically components [3].

Then the pdf of $X_{(I: n)}, 1 \leq i \leq n$ is given by

$f_{i: n}=\frac{1}{B(i, n-i+1)}[F(x)]^{i-1}[1-F(x)]^{n-i} f(x)$

Then by using $\operatorname{Eq}(10)$ and $\operatorname{Eq}(11)$, we get

$$
\begin{aligned}
& f_{i: n}(\mathbf{x})
\end{aligned}
$$

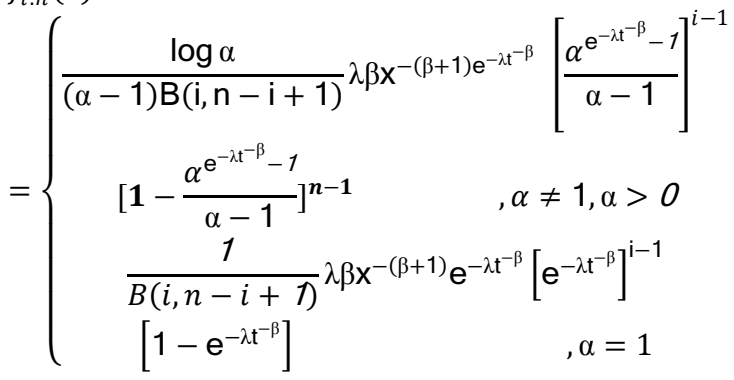

We define the first order statistics $X_{(1)}=\min \left(X_{1}, X_{2}, \ldots . . X_{n}\right)$ the last order statistics $X_{(n)}=$ $\max \left(X_{1}, X_{2}, \ldots . X_{n}\right)$.

\subsection{Distribution of minimum, maximum and median}

Let $X_{1}, X_{2}, \ldots, X_{n}$ be independent, identically distributed random variables from APIW.

$$
\begin{aligned}
& f_{\text {max }}(x)=\frac{d}{d x}(F(x))^{n}=n(F(x))^{n-1} f(x) \\
& =\left\{\begin{array}{c}
\frac{n \log \alpha}{(\alpha-1)^{n}} \lambda \beta x^{-(\beta+1)} \mathrm{e}^{-\lambda \mathrm{t}^{-\beta}} \alpha^{\mathrm{e}^{-\lambda \mathrm{t}-\beta}} \\
{\left[\alpha^{\left.\mathrm{e}^{-\lambda \mathrm{t}^{-\beta}}-1\right]^{\alpha-1}}, \alpha>0, \alpha \neq 1\right.} \\
n \lambda \beta x^{-(\beta+1)} \mathrm{e}^{-\lambda \mathrm{t}^{-\beta}\left[\mathrm{e}^{-\lambda \mathrm{t}-\beta}\right]^{n-1}}, \alpha=1
\end{array}\right. \\
& f_{\text {min }}(x)=-\frac{d}{d x}(1-F(x))^{n}=n(1-F(x))^{n-1} f(x) \\
& =\left\{\begin{array}{cc}
\frac{\log \alpha}{(\alpha-1)^{n}} \lambda \beta x^{-(\beta+1)} e^{-\lambda x^{-\beta}} \alpha^{e^{-\lambda x^{-\beta}}} & \\
{\left[\alpha-\alpha^{e^{-\lambda x^{-\beta}}}\right]^{n-1}} & , \alpha>0, \alpha \neq 1 \\
n \lambda \beta\left(1-e^{-\lambda x^{-\beta}}\right)^{n-1} x^{-(\beta+1) e^{-\lambda x^{-\beta}}} \quad, \alpha=1
\end{array}\right.
\end{aligned}
$$

and

$$
\begin{gathered}
f_{\text {med }}(x)=\frac{(2 m+1) !}{m ! m !}[F(x)]^{m}[1-F(x)]^{m} f(x) \\
=\left\{\begin{array}{c}
\frac{(2 m+1) !}{m ! m !} \frac{\lambda \beta \log \alpha}{(\alpha-1)^{2 m}} x^{-(\beta+1)} e^{-\lambda x^{-\beta}} \alpha^{e^{-\lambda x}-\beta} \\
{\left[\alpha^{\left.e^{-\lambda x^{-\beta}}-1\right]^{m}\left[\alpha-\alpha^{e^{-\lambda x^{-\beta}}}\right]^{m},}, \alpha>0, \alpha \neq 1\right.} \\
\frac{(2 m+1)}{m ! m !} \lambda \beta x^{-(\beta+1)}\left[e^{-\lambda x^{-\beta}}\right]^{m+1} \\
{\left[1-e^{-\lambda x^{-\beta}}\right]^{m} \quad, \alpha=1}
\end{array}\right.
\end{gathered}
$$

\section{MAXIMUM LIKLIHOOD} ESTIMATES

In this section, we derive the maximum likelihood estimates of the unknown parameters $\alpha, \beta$, and $\lambda$ of $\operatorname{APIW}(\alpha, \beta, \lambda)$ based on a complete sample. 
Let $X_{1}, X_{2}, \ldots, X_{n}$ be a random sample from $\operatorname{APIW}(\alpha, \beta$,$) then$ the log-likelihood function can be obtained as

$$
\begin{gathered}
L(\alpha, \beta, \lambda)=n \ln (\ln \alpha)-n \ln (\alpha-1)+n \ln (\lambda \beta)-(\beta \\
+1) \\
\sum_{i=1}^{n} \log \left(x_{i}\right)-\lambda \sum_{i=1}^{n} x_{i}^{-\beta}+\log (\alpha) \sum_{i=1}^{n} e^{-\lambda x^{-\beta}} .
\end{gathered}
$$

The normal equation can be obtained by taking the first partial derivatives of the log-likelihood function with respect to the three parameters and equating the first derivative equal zero we get the following normal equations:

$$
\begin{gathered}
\frac{n}{\alpha \ln \alpha}-\frac{n}{\alpha-1}=0 \\
\frac{n}{\lambda}-\sum_{i=1}^{n} x_{i}^{-\beta}+\ln (\alpha) \sum_{i=1}^{n} x_{i}^{-\beta} e^{-\lambda x^{-\beta}}=0
\end{gathered}
$$

and

$$
\frac{n}{\beta}-\sum_{i=1}^{n} \ln \left(x_{i}\right)+\lambda \ln (\alpha) \sum_{i=1}^{n} x_{i}^{-\beta} \ln \left(x_{i}\right) e^{-\lambda x^{-\beta}}=0
$$

The normal equations do not have explicit solutions and they have to be obtained numerically.

\subsection{Asymptotic Confidence Bounds}

As the MLEs of the unknown parameters $\alpha, \beta$ and $\lambda$ cannot be obtained in closed forms, it is difficult to derive the exact distributions of the MLEs. In this section, we derive the asymptotic confidence intervals of these parameters when $\alpha>0, \beta>0$ and $\lambda>0$. The simplest large sample approach is to assume that $\operatorname{MLE}(\hat{\alpha}, \hat{\beta}, \hat{\lambda})$ are approximately multivariate normal with mean $(\alpha, \beta, \lambda)$ and covariance $I_{0}^{-1}$ matrix $I_{O}^{-1}$, see Lawless [8], in which is the inverse of the observed information matrix

$I_{O}^{-1}=\left(\begin{array}{ccc}-\frac{\partial^{2} \mathrm{~L}}{\partial \lambda^{2}} & -\frac{\partial^{2} \mathrm{~L}}{\partial \lambda \partial \beta} & -\frac{\partial^{2} \mathrm{~L}}{\partial \lambda \partial \alpha} \\ -\frac{\partial^{2} \mathrm{~L}}{\partial \beta \partial \lambda} & -\frac{\partial^{2} \mathrm{~L}}{\partial \beta^{2}} & -\frac{\partial^{2} \mathrm{~L}}{\partial \beta \partial \alpha} \\ -\frac{\partial^{2} \mathrm{~L}}{\partial \alpha \partial \lambda} & -\frac{\partial^{2} \mathrm{~L}}{\partial \alpha \partial \beta} & -\frac{\partial^{2} \mathrm{~L}}{\partial \alpha^{2}}\end{array}\right)$

where,

$$
\begin{aligned}
& \frac{\partial^{2} \mathrm{~L}}{\partial \lambda^{2}}=-\frac{\mathrm{n}}{\alpha^{2} \log \alpha}-\frac{\mathrm{n}}{\alpha^{2} \log \alpha^{2}}+\frac{\mathrm{n}}{(\alpha-1)^{2}}-\sum_{\mathrm{i}=1}^{\mathrm{n}} \frac{e^{-\lambda x^{-\beta}}}{\alpha^{2}}, \\
& \frac{\partial^{2} \mathrm{~L}}{\partial \lambda \partial \beta}=-\frac{\mathrm{n}}{\beta^{2}}-\lambda \sum_{\mathrm{i}=1}^{\mathrm{n}} \mathrm{x}_{\mathrm{i}}^{-\beta}\left(\log \mathrm{x}_{\mathrm{i}}\right)^{2} \\
& +\lambda \log (\alpha) \sum_{\mathrm{i}=1}^{\mathrm{n}}\left[\lambda \mathrm{x}_{\mathrm{i}}^{-\beta}-1\right] \mathrm{x}_{\mathrm{i}}^{-\beta}\left(\log \mathrm{x}_{\mathrm{i}}\right)^{2} e^{-\lambda x^{-\beta}}, \\
& \frac{\partial^{2} \mathrm{I}}{\partial \lambda^{2}}=-\frac{\mathrm{n}}{\lambda^{2}}+\log (\alpha) \sum_{\mathrm{i}=1}^{\mathrm{n}}\left(\mathrm{x}_{\mathrm{i}}^{\beta}\right)^{2} e^{-\lambda x^{-\beta}}, \\
& \frac{\partial^{2} \mathrm{~L}}{\partial \beta \partial \lambda}=\sum_{i=1}^{n} \mathrm{x}_{\mathrm{i}}^{-\beta} \log \left(\mathrm{x}_{\mathrm{i}}\right)
\end{aligned}
$$

$+\log (\alpha) \sum_{i=1}^{n}\left[1-\lambda x_{i}^{-\beta}\right] x_{i}^{-\beta} \log \left(x_{i}\right) e^{-\lambda x^{-\beta}}$,

$\frac{\partial^{2} L}{\partial \alpha \partial \lambda}=-\sum_{i=1}^{n} \frac{x_{i}^{-\beta} e^{-\lambda x^{-\beta}}}{\alpha}$

and

$\frac{\partial^{2} \mathrm{~L}}{\partial \alpha \partial \beta}=\sum_{\mathrm{i}=1}^{\mathrm{n}} \frac{\lambda \mathrm{x}_{\mathrm{i}}^{-\beta} \log \left(X_{i}\right) e^{-\lambda x^{-\beta}}}{\alpha}$.

The above approach is used to derive the $100(1-\Upsilon) \%$ confidence intervals for the parameters $\alpha$, $\beta$ and $\lambda$ are determined respectively as

$\hat{\alpha} \pm Z_{\gamma / 2} \sqrt{\operatorname{Var}(\hat{\alpha})}, \hat{\lambda} \pm Z_{\gamma / 2} \sqrt{\operatorname{Var}(\hat{\lambda})}$ and $\hat{\beta} \pm Z_{\gamma / 2} \sqrt{\operatorname{Var}(\hat{\beta})}$.

where $\operatorname{Var}(\widehat{\alpha},) \operatorname{Var}(\hat{\beta})$ and $\operatorname{Var}(\hat{\lambda})$ are the variance of $\hat{\alpha}, \hat{\beta}$ and $\hat{\lambda}$, which are given by the diagonal elements of $I_{O}^{-1}$ and $Z_{\frac{Y}{2}}$ is the upper $\frac{r}{2}$ percentile of the standard normal distribution.

\section{SIMULATION STUDIES}

The Equation $F(x)-u=0$, where $\mathrm{u}$ is an observation from the uniform distribution on $(0,1)$ and $F(x)$ is cumulative distribution function of the distribution is used to carry out the simulation study to generate data from distribution.

The simulation experiment was repeated $N=1000$ times each with sample sizes; $n=(20,30,50,70)$ and $(\alpha, \beta, \lambda)=$ $(10,1.5,0.5),(5,2.5,0.5)$. The following measures are computed.

Average bias and the mean square error (MSE) of $\hat{\theta}$ of the parameter $\alpha, \beta$, and $\lambda$

$$
\frac{1}{N} \sum_{i=1}^{n}(\hat{\theta}-\theta) \text { and } \frac{1}{N} \sum_{i=1}^{n}(\widehat{\theta}-\theta)^{2} .
$$

Table 1 presents the average bias and the MSE of the estimates. The values of the bias and the MSEs decreases while the sample size increase

Table 1. presents the average bias and the MSE of the estimates. The values of the bias and the MSEs decreases while the sample size increases

\begin{tabular}{|l|l|l|l|l|l|l|l|l|l|}
\hline$\alpha$ & $\beta$ & $\lambda$ & $\mathrm{n}$ & $\begin{array}{l}\text { Bias } \\
(\alpha)\end{array}$ & $\begin{array}{l}\text { MSE } \\
(\alpha)\end{array}$ & $\begin{array}{l}\text { Bias } \\
(\beta)\end{array}$ & $\begin{array}{l}\text { MSE } \\
(\beta)\end{array}$ & $\begin{array}{l}\text { Bia } \\
\text { s } \\
(\lambda)\end{array}$ & $\begin{array}{l}\text { MS } \\
(\lambda)\end{array}$ \\
\hline 10 & 1.5 & .5 & 20 & -9.08 & 84.4 & -0.12 & 0.29 & 0.56 & 0.36 \\
\hline & & & 30 & -9.11 & 83.2 & -0.16 & 0.12 & 0.54 & 0.35 \\
\hline & & & 50 & -9.18 & 80.6 & -0.22 & 0.10 & 0.40 & 0.31 \\
\hline & & & 70 & -9.24 & 78.7 & -0.29 & 0.08 & 0.40 & 0.30 \\
\hline 5 & 2.5 & .5 & 20 & -7.21 & 53.0 & -0.20 & 0.11 & 0.49 & 0.22 \\
\hline & & & 30 & -7.22 & 51.8 & -0.23 & 0.10 & 0.40 & 0.21 \\
\hline & & & 50 & -7.25 & 46.8 & -0.24 & 0.1 & 0.38 & 0.20 \\
\hline & & & 70 & -7.31 & 39.7 & -0.26 & 0.09 & 0.35 & 0.19 \\
\hline
\end{tabular}




\section{APPLICATION}

Here, the applicability of the APIW distribution is illustrated using real data sets from [5] the data set consist of 72 observation.

$\begin{array}{lllllllllllllll}12 & 15 & 22 & 24 & 24 & 32 & 32 & 33 & 34 & 38 & 38 & 43 & 44 & 48 & 52\end{array}$

$\begin{array}{lllllllllllllll}53 & 54 & 54 & 55 & 56 & 57 & 58 & 58 & 59 & 60 & 60 & 60 & 60 & 61 & 62\end{array}$

$\begin{array}{lllllllllllllll}63 & 65 & 65 & 67 & 68 & 70 & 70 & 72 & 73 & 75 & 76 & 76 & 81 & 83 & 84\end{array}$

$\begin{array}{lllllllllllll}85 & 87 & 91 & 95 & 96 & 98 & 99 & 109 & 110 & 121 & 127 & 129 & 131\end{array}$

$\begin{array}{lllllllllll}143 & 146 & 146 & 175 & 175 & 211 & 233 & 258 & 258 & 263 & 341\end{array}$ 341376

We compare the fit of the APIW distribution with those of the inverse Weibull, Frechet, inverse Raylight and inverse Exponential distributions. The model selection is carried out using the AIC (Akaike information criterion), the BIC (Bayesian information criterion), the CAIC (consistent Akaike information criteria) and the HQIC (Hannan Quinn information criterion).

Table2: Estimated parameters, AIC, CAIC, BIC and HOIC for the real data set

\begin{tabular}{|c|c|c|c|c|c|c|c|c|}
\hline \multirow[t]{2}{*}{ Dis. } & \multicolumn{3}{|c|}{ Parameters } & \multirow[t]{2}{*}{$-2 l$} & \multirow{2}{*}{$\begin{array}{l}\text { AI } \\
\text { C }\end{array}$} & \multirow{2}{*}{$\begin{array}{l}\text { CA } \\
\text { IC }\end{array}$} & \multirow{2}{*}{$\begin{array}{l}\text { BI } \\
\mathrm{C}\end{array}$} & \multirow{2}{*}{$\begin{array}{l}\text { HQ } \\
\text { IC }\end{array}$} \\
\hline & $\widehat{\alpha}$ & $\widehat{R}$ & $\hat{\lambda}$ & & & & & \\
\hline $\begin{array}{l}\text { APIW } \\
(\alpha, \beta, \lambda)\end{array}$ & 99.16 & 1.78 & 343.8 & 391.1 & 788. & 388.7 & 795.1 & 392.7 \\
\hline $\begin{array}{l}\mathrm{IW} \\
(\beta, \lambda)\end{array}$ & & 1.41 & 284.0 & 791.2 & 795. & 795.4 & 799.8 & 797.1 \\
\hline $\mathrm{F}(\beta)$ & & 0.31 & & 1026. & 1028 & 1028. & 1030. & 1029 \\
\hline $\operatorname{IR}(\boldsymbol{\lambda})$ & & & 2187 & 813.4 & 815. & 815.5 & 817.7 & 816.3 \\
\hline $\operatorname{If}(\boldsymbol{\lambda})$ & & & 60.09 & 805.3 & 805. & 807.4 & 809.6 & 808.2 \\
\hline
\end{tabular}

The maximum likelihood estimates and the corresponding AIC, CAIC, BIC, and HQIC values are shown in Table 2. We can see that the smallest AIC, CAIC, BIC, and HQIC are obtained for the APIW distribution. So, one can conclude that the APIW distribution is the most appropriate model for this data set among the considered distributions.

Now, we shall apply formal goodness-of-.t-tests in order to verify which distribution fits better these real data sets. We consider the Kolmogorov.

Smirnov k-s, P-value, Anderson. Darling A and Watson W statistics, which are described in details by [6]. In general, the smaller the values of these statistics, the better the .t to the data. The statistics k-s, P-value, W and A for all the models are listed in Table 3. The APIW distribution approximately provides an adequate fit for the data

Table3: Goodness-of-fit statistics corresponds to data set

\begin{tabular}{|l|l|c|l|l|}
\hline Dis. & $\mathrm{k}-S$ & $\mathrm{p}-$ value & $\mathrm{A}$ & $\mathrm{W}$ \\
\hline APIW & 0.109619 & 0.35247 & 0.687468 & 0.111395 \\
\hline IW & 0.1381 & 0.1381 & 1.5177 & 0.2538 \\
\hline $\mathrm{F}$ & 0.6398 & $8.95 \times 10^{-27}$ & 44.042 & 9.6086 \\
\hline IR & 0.2369 & $4.88 \times 10^{-4}$ & 6.5639 & 1.2661 \\
\hline IF & 0.1846 & 0.0126 & 4.6425 & 0.8379 \\
\hline
\end{tabular}

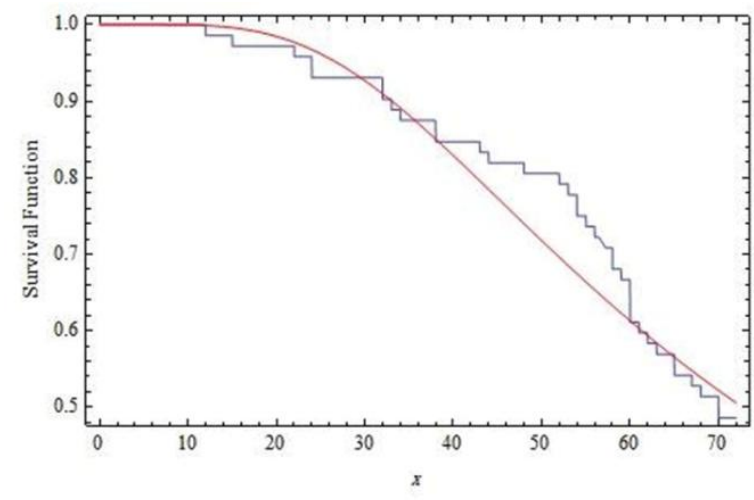

Fig.5. The empirical and fitted cumulative for the APIW

\section{CONCLUSION}

In this paper, a three parameters alpha power inverse Weibull distribution have introduced, based on alpha-power transformation method (APT). Most statistical and reliability properties are derived and studied. Simulation schemes are formulated and provide less bias and mean square error as a sample size increases for MLEs of APIW parameters. Point Estimation via MLE method is done moreover, the Fisher information matrix for interval estimation is studied for APIW. A real data is used to illustrate and compare the potential of APIW distribution with other competing distributions showed that it could offer a better fit than a set of extensions of Weibull distribution.

\section{ACKNOWLEDGMENTS}

The authors thank the referees and editor for their valuable comments and suggestions for improving the paper. Our thanks to the experts who have contributed towards the development of the template.

\section{REFERENCES}

[1] Abbas, K. D. 2017. A new method for generating distributions with an application to exponential distribution." s.l, s.n, 46, 1-25.

[2] Abdelrahman, N. S. and El-Bassiouny, A.H. 2017, On the alpha-power exponential weibull distribution, The 2nd National of Mathematics and Its Applications (NCMA17), 1-18.

[3] Arnold, B. C. N. B. H. N. 2008. A .first course in order statistics .John Wiley and Sons, New York, 54, 9-12.

[4] Bain, L.J. 1974, Analysis for the linear failure-rate lifetesting distribution, New York, 13, 551-559.

[5] Bjerkedal, T. 1960. Acquisition of resistance in guinea pigs infected with different doses of virulent tubercle bacilli". American Journal of Epidemiology, 72, 130148.

[6] Chen, G. and Balakrishnan, C. 1995, A general purpose approximate goodness-of-.t test, Journal of Quality Technolog, 27, 154.161.

[7] Corderio, M.G. and Lemonte, JA. 2013, On the Marshal Olkin extended weibull distribution, State, 54, 333-353

[8] Lawless, J. F. 2003, Statistical models and methods for lifetime data, John Wiley and Sons, 362, 8-14 New York.

[9] Mahdavi, A and Kundu, D. 2015, A new method for generating distributions with an application to 
International Journal of Computer Applications (0975 - 8887)

Volume 181 - No. 11, August 2018

exponential distribution, Communications in StatisticsTheory and Methods, 46(13), 6543-6557.

[10] Nassar, M and Abo-Kasem, O. E. 2017, Estimation of the inverse Weibull parameters under adaptive type-II progressive hybrid censoring scheme, Journal of Computational and Applied Mathematics, 315, 228-239.

[11] Sarhan, A. M and Apaloo, J. 2013. Exponentiated modified weibull extension distribution. Reliability Engineering and System Safety, 112, 137-144. 